\title{
Within-Laboratory Variance Outlier Detection: An Alternative to Cochran's Test *
}

\author{
by
}

Michael J. Morton

Altria Client Services LLC, 601 E. Jackson, Richmond, Virginia 23219, USA

\section{SUMMARY}

An important step in the full definition of an analytical method is the characterization of the within and between laboratory variability. This is typically done through collaborative studies involving multiple laboratories. The statistical analysis of the results of collaborative studies is generally carried out using standardized protocols such as those given in ISO 5725-2 or ASTM E691-14.

One aspect of the evaluation of collaborative studies is the identification of outlying laboratories which are then excluded from the variance calculation associated with the analytical method. Whether particular laboratories are identified as outliers can have a dramatic effect on the calculated variances.

The generally recommended approach to identify laboratories with excessive within-laboratory variation is Cochran's Test or something similar. However, Cochran's Test is very sensitive to non-normality of the underlying statistical distribution. When the assumption of normality is violated, Cochran's Test can wrongly identify laboratories as outliers at much greater than the nominally stated error rate, even for deviations from normality that are very difficult to detect analytically.

In this paper, an alternative to Cochran's Test, adapted from Levene's Test, is proposed and shown to approximately maintain the stated error rate when the underlying distribution is not normal. This newly adapted test is recommended for future collaborative study analysis in place of Cochran's Test. [Beitr. Tabakforsch. Int. 27 (2017) 135-144]

\section{KEYWORDS}

Collaborative study, outlier identification, homogeneity of variance test, Cochran's Test, Levene's Test, BrownForsythe Test, k-statistic.

\section{ZUSAMMENFASSUNG}

Ein wichtiger Schritt bei der vollständigen Definition einer Analysemethode ist die Charakterisierung der Variabilität innerhalb und zwischen Laboren. Dies erfolgt in der Regel durch gemeinschaftliche Studien unter Beteiligung mehrerer Labore. Die statistische Auswertung der Ergebnisse solcher Studien wird im Allgemeinen mit standardisierten Protokollen wie in ISO 5725-2 oder ASTM E691-14 vorgenommen.

Ein Aspekt der Auswertung von gemeinschaftlichen Studien ist die Identifizierung von Ausreißer-Laboren, die dann von der Berechnung der Varianz im Zusammenhang mit der Analysemethode ausgeschlossen werden. Ob bestimmte Labore als Ausreißer erkannt werden, kann erhebliche Auswirkungen auf die berechneten Varianzen haben.

Der allgemein empfohlene Ansatz für die Identifikation von Laboren mit einer übermäßig hohen Variation innerhalb des jeweiligen Labors ist der Cochran-Test oder Ähnliches. Der Cochran-Test reagiert jedoch sehr empfindlich auf die Nicht-Normalität der zugrundeliegenden statistischen Verteilung. Wird die Annahme der Normalität verletzt, kann der Cochran-Test mit einer viel höheren als der nominal angegeben Fehlerquote fälschlicherweise Labore als Aus- 
reißer erkennen, und das selbst für Abweichungen von der Normalität, die analytisch sehr schwer festzustellen sind. In dieser Arbeit wird eine Alternative für den Cochran-Test vorgeschlagen, die eine auf dem Levene-Test basiert. Es wird gezeigt, dass damit die angegebene Fehlerquote ungefähr gleich bleibt, wenn die zugrundeliegende Verteilung nicht normal ist. Für zukünftige gemeinschaftliche Studienanalysen wird dieser neu adaptierte Test anstelle des Cochran-Tests empfohlen. [Beitr. Tabakforsch. Int. 27 (2017) 135-144]

\section{RESUME}

Une étape importante vers la définition complète d'une méthode analytique est la caractérisation de la variabilité inter- et intra-laboratoire. En règle générale, cette caractérisation passe par des études collaboratives impliquant de multiples laboratoires. Généralement, l'analyse statistique des études collaboratives est réalisée au travers de protocoles normalisés tels que ceux proposés dans les normes ISO 5725-2 ou ASTM E691-14.

Une facette de l'évaluation des études collaboratives porte sur l'identification des laboratoires dits aberrants, qui sont alors exclus du calcul de variance associé à la méthode analytique. L'identification de laboratoires particuliers parmi les données aberrantes peut avoir un effet notable sur les variances calculées.

L'approche communément préconisée en vue d'identifier les labos présentant une variation intra-laboratoire excessive est le test de Cochran ou toute autre épreuve similaire. Toutefois, le test de Cochran s'avère très sensible à toute anomalie de la distribution statistique sous-jacente. Si la présomption de normalité est enfreinte, le test de Cochran peut erronément classer des laboratoires parmi les données aberrantes, avec un taux d'erreurs nettement plus élevé que le taux nominal, même dans le cas d'écarts de normalité qui sont très difficiles à détecter par voie analytique.

Le présent article propose une option alternative au test de Cochran, adaptée du test de Levene et démontre que cette proposition conserve approximativement le taux d'erreur indiqué lorsque la distribution sous-jacente présente des anomalies. Ce test récemment adapté est recommandé pour les futures analyses collaboratives en remplacement du test de Cochran. [Beitr. Tabakforsch. Int. 27 (2017) 135-144]

\section{INTRODUCTION}

An important part of the characterization of an analytical method is the estimation of the variability associated with the method, both within a laboratory and across laboratories. Those estimated variances can be strongly influenced by the values that are identified as outliers, since the variation that is calculated may be substantially different depending on whether a laboratory or individual data point is or is not identified as an outlier.

One of the criteria typically applied to the determination of outlying laboratories in a collaborative study relates to the level of replicate-to-replicate variability within individual laboratories. ISO 5725-2 (1) recommends the use of Cochran's Test at the $1 \%$ level for the determination of outlying laboratories. ASTM E691-14 (2) recommends the use of the k-statistic (sometimes called Mandel's k) at the nominal $\alpha$-level of $0.5 \%$. The two statistics are statistically equivalent, since $k=\sqrt{p c}$, where $p$ is the number of laboratories in the collaborative study and $c$ is the Cochran statistic. However, somewhat confusingly, the stated $\alpha$-level of Cochran's Test conventionally makes allowance for multiple testing, whereas the k-statistic does not. Thus the stated $0.5 \%$ level in the ASTM E691-14 recommendation is quite a bit more liberal than the stated $1 \%$ level in ISO $5725-2$, since the actual level of the k-statistic is approximately $p \times 0.5 \%$.

Additionally, some practitioners follow the protocol "Harmonized statistical procedure" defined by IUPAC (International Union of Pure and Applied Chemistry) (3). The procedure consists of sequential application of the Cochran and Grubbs tests at a 2.5\% level until no further outliers are detected or until $22.2 \%$ of the laboratories are deleted.

It has long been known that the F-statistic used in testing whether variances are the same in two populations is quite sensitive to deviations of the underlying populations from normality (4). Since Cochran's Test and the k-statistic are derived from the F-Test, it would be expected that they are similarly or even more sensitive to deviations from normality and this was confirmed by CONOVER et al. (5).

Several approaches that are less subject to the sensitivity to deviations from normality seen with the F-test, Cochran's Test, or the k-statistic have been proposed for the general problem of testing whether all of the within-group-variances are the same (for example, see (6) and (7)). However, in the case of a collaborative study, one is testing whether one or more laboratories have excessively large variations, not simply whether there are differences among the withinlaboratory variances and none of the proposed alternative approaches addresses the specific problem of interest in a collaborative study.

A class of alternative approaches to testing whether all group variances are the same is based on proposals originally made by LEVENE (6) with additional modifications by BROWN and FORSYTHE (7). In this paper, those approaches are adapted to specifically address whether a laboratory has excessive within-laboratory variation.

\section{METHODS}

Typically in a collaborative study, several laboratories analyze a set of matched samples for one or more analytes. For simplicity of notation, focus on one matched sample going to each laboratory with one measured analyte. Each laboratory then analyzes the sample with multiple replicates. Having excessively large within-laboratory variation is one of the criteria for eliminating a laboratory from the analysis. That is, the outlier test establishes criteria by which the replicate-to-replicate variation of a laboratory is declared to be "too large" and the laboratory is dropped from the analysis.

\subsection{Cochran's Test}

ISO 5725-2 recommends the use of Cochran's Test at the $1 \%$ significance level to determine whether any of the 
laboratories have excessively large variations. Denoting each of the within-laboratory sample standard deviations by $s_{i}$, with $i$ indicating the $i$-th of the $p$ laboratories, Cochran's Test consists of forming the ratio:

$$
c=\frac{s_{\max }^{2}}{\sum_{i=1}^{p} s_{i}^{2}}
$$

where $s_{\max }$ is the largest within-laboratory standard deviation. The laboratory with the largest sample standard deviation is then removed as an outlier if $c$ exceeds the $1 \%$ critical value. If a laboratory is eliminated as an outlier, it is common to then repeat Cochran's Test on the remaining $p-1$ laboratories and to continue until Cochran's Test does not show statistical significance.

\section{2. $k$-Statistic}

ASTM E691-14 recommends the use of a similar statistic given by

$$
k=\sqrt{\frac{s_{\max }^{2}}{\sum_{i=1}^{p} s_{i}^{2} / p}}
$$

In ISO 5725-2, the k-statistic is denoted as Mandel's k. It can be seen that $k=\sqrt{p c}$, and thus Cochran's Test and the k-statistic would be statistically equivalent if the critical values were both calculated similarly. ASTM E691-14 recommends using a nominal $0.5 \%$ level of significance for $k$. Since the nominal level of significance is smaller, one would think that the ASTM E691-14 approach is more conservative than the one recommended by ISO $5725-2$, since $0.5 \%<1 \%$. However, the two standards refer to significance levels differently in a manner that is likely to be confusing to many practitioners. The critical values associated with the k-statistic do not correct for multiple testing, whereas the tables associated with Cochran's Test incorporate a Bonferroni correction for multiple testing.

For example, suppose there are $p=10$ laboratories in a collaborative study and each laboratory carries out $r=3$ replicates. Under normality Cochran's Test is derived to only falsely claim one of the laboratories to have excessive variation approximately $1 \%$ of the time. The k-statistic is built to only falsely claim that a single pre-specified laboratory (for example laboratory \#1) has excessive variation $0.5 \%$ of the time. However, since each laboratory has $0.5 \%$ chance to be falsely identified, cumulatively, across all 10 laboratories, there is approximately a $10 \times 0.5 \%=5 \%$ chance that one or more laboratories will be falsely identified. So, in this instance, the ASTM E691-14 0.5\%-criterion for identifying outliers is equivalent to the ISO $5725-2$ $5 \%$-criterion for identifying stragglers using Cochran's Test. In general the actual level of significance associated with the ASTM E691-14 recommended outlier test will be approximately $0.5 \% \times p$, where, again, $p$ is the number of laboratories in the collaborative study.
To illustrate the discussion above, assuming a collaborative study with three replicates from each of ten laboratories, note that the 5\% value for Cochran's Test in Table 4 of ISO $5725-2\left(c_{c r i t}=0.445\right)$ can be used to calculate the $0.5 \%$ point in Table 5 of ASTM E691-14 $\left(k_{\text {crit }}=2.11\right)$, using the relationship between $k$ and $c$ noted above,

$2.11=k_{\text {crit }}=\sqrt{p c_{\text {crit }}}=\sqrt{10 \times 0.445}$.

\subsection{Levene's Test}

As noted above, LEVENE (6) proposed an alternative approach to comparing within group variances using the idea that the analysis of variance is robust to deviations from normality. Focusing on a single laboratory to simplify the notation, the idea is to replace each of the raw data values $x_{i}$ with some function of differences $D_{i}=\left|x_{i}-\tilde{X}\right|$, where $\tilde{x}$ is typically either the mean or median of the replicates. An analysis of variance is then carried out across the laboratories on the $D_{i}$ with statistical significance indicating that there are one or more differences among the within-laboratory variances. More references and further discussion of the history of this approach can be found in GASTWIRTH et al. (8).

CONOVER et al. (5) and BROWN and FORSYTHE (7) reported simulation results of a variety of approaches consistent with Levene's original idea. However, the simulations did not include the numbers of groups (i.e., number of laboratories) typically seen in collaborative studies. Therefore simulations covering the number of laboratories often seen in collaborative studies were conducted to determine which version of Levene's test to adapt as an alternative to Cochran's Test. The following versions of Levene's Test are considered:

1. L2, carrying out the analysis on $\mathrm{D}_{\mathrm{i}}^{2}$ with $\tilde{x}$ defined to be the mean.

2. L1, carrying out the analysis on the $\mathrm{D}_{\mathrm{i}}$ with $\tilde{x}$ defined to be the mean.

3. Lmed, carrying out the analysis on the $\mathrm{D}_{\mathrm{i}}$ with $\tilde{x}$ defined to be the median

4. L0, which is the same as Lmed except when the number of replicates is odd (making the median one of the data points), the $D_{i}$ associated with the median of the $x_{i}$ is dropped for the purposes of the outlier test. The deleted value is always equal to zero and is sometimes referred to as a "structural zero" (9).

The first three versions, L2, L1, and Lmed, are the most commonly implemented. Lmed is often referred to as the Brown-Forsythe Test. The fourth, L0, was suggested in CONOVER et al. (5) and examined in HINES and HINES (9).

\subsection{Non-normal distributions}

The concern with Cochran's Test relates to its sensitivity to deviations of the underlying distribution from normality. The deviations from normality considered here are:

1. "Heavy-tailed" distributions, illustrated by a t-distribution with four degrees of freedom;

2. Skewed distributions, illustrated by an exponential distribution.

Figure 1 shows the "heavy-tailed" alternative plotted together with the normal distribution. It is unlikely that many 


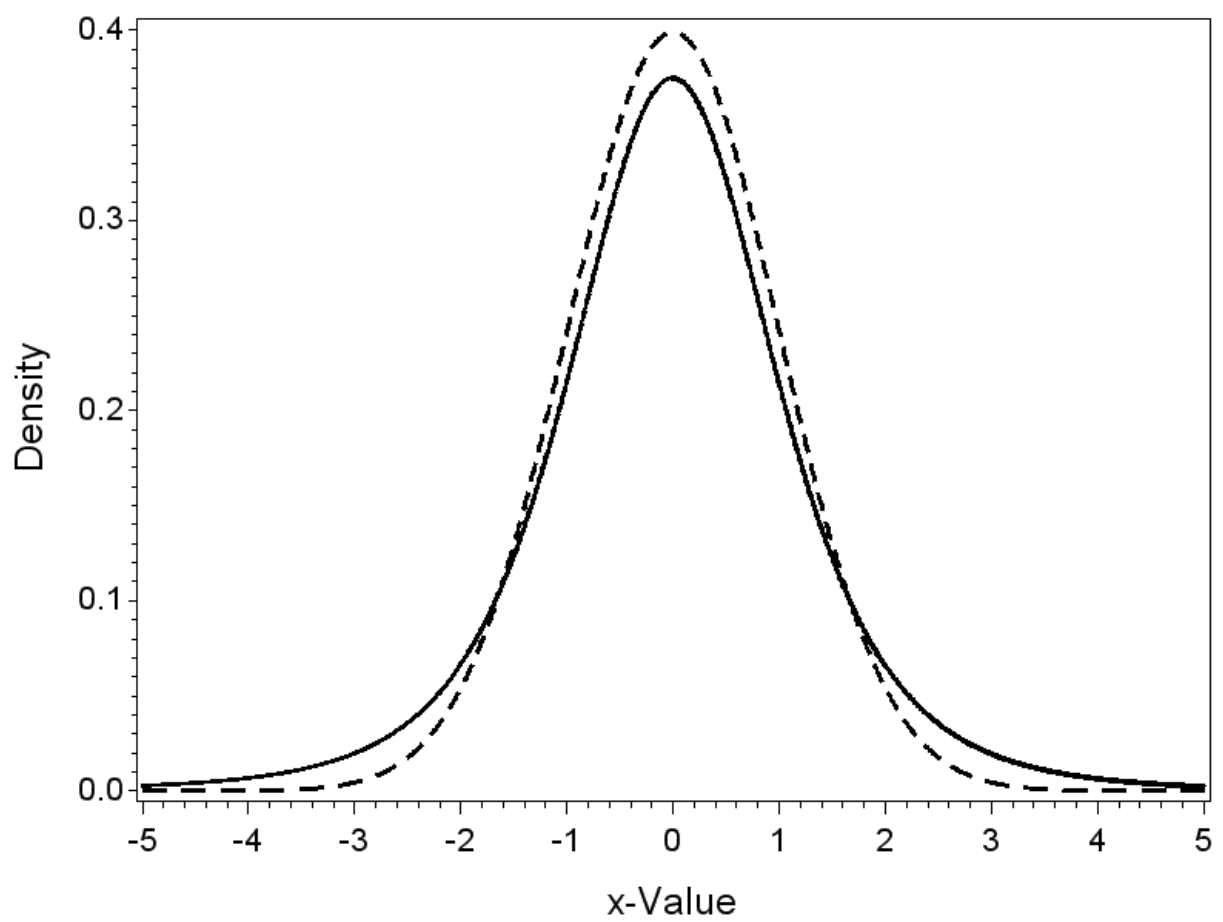

Figure 1. Heavy-tailed distribution (t with four degrees of freedom) plotted with the solid line and, for reference, a normal distribution plotted with a dashed line.

observers seeing the "heavy-tailed" alternative in isolation would have any concerns because it looks relatively "bellshaped" and would be difficult to distinguish from a normal distribution.

Table 1 shows simulations to determine the power of two standard tests for normality $(10,11)$ against the two non- normal distributions considered here. The table shows that it is very difficult to determine that the "heavy-tailed" alternative is non-normal, certainly requiring $n>100$ to reliably distinguish it from a normal distribution. Not surprisingly, it is much easier to determine that values from the skewed distribution are not normally distributed.

Table 1. Estimated probability to detect deviation from normality. ${ }^{a}$

\begin{tabular}{|c|c|c|c|c|}
\hline \multirow{2}{*}{ Sample size } & \multicolumn{2}{|c|}{ Heavy-tailed distribution ${ }^{b}$} & \multicolumn{2}{|c|}{ Skewed distribution $^{\mathrm{C}}$} \\
\hline & Shapiro-Wilk Test & Anderson-Darling Test & Shapiro-Wilk Test & Anderson-Darling Test \\
\hline 10 & $12 \%$ & $13 \%$ & $46 \%$ & $43 \%$ \\
\hline 20 & $24 \%$ & $23 \%$ & $85 \%$ & $79 \%$ \\
\hline 30 & $33 \%$ & $30 \%$ & $97 \%$ & $94 \%$ \\
\hline 40 & $39 \%$ & $35 \%$ & $100 \%$ & $99 \%$ \\
\hline 50 & $45 \%$ & $41 \%$ & $100 \%$ & $100 \%$ \\
\hline 60 & $52 \%$ & $48 \%$ & $100 \%$ & $100 \%$ \\
\hline 70 & $55 \%$ & $50 \%$ & & \\
\hline 80 & $62 \%$ & $56 \%$ & & \\
\hline 90 & $65 \%$ & $60 \%$ & & \\
\hline 100 & $68 \%$ & $62 \%$ & & \\
\hline 110 & $74 \%$ & $68 \%$ & & \\
\hline 120 & $77 \%$ & $72 \%$ & & \\
\hline 130 & $81 \%$ & $75 \%$ & & \\
\hline 140 & $83 \%$ & $78 \%$ & & \\
\hline 150 & $85 \%$ & $80 \%$ & & \\
\hline 160 & $88 \%$ & $83 \%$ & & \\
\hline 170 & $88 \%$ & $83 \%$ & & \\
\hline 180 & $91 \%$ & $87 \%$ & & \\
\hline 190 & $90 \%$ & $88 \%$ & & \\
\hline 200 & $93 \%$ & $90 \%$ & & \\
\hline
\end{tabular}

a Probability is estimated from $n=1000$ simulation runs

b Heavy-tailed distribution is represented by $t$ with 4 degrees of freedom Skewed distribution is represented by an exponential distribution 
Table 2. Simulation ( $n=10,000$ realizations) to determine the sensitivity of Cochran's Test and the k-test to deviations from normality.

\begin{tabular}{|c|c|c|c|c|c|c|c|}
\hline \multirow{2}{*}{$\begin{array}{l}\text { Number of } \\
\text { Labs }\end{array}$} & \multirow[b]{2}{*}{ Reps } & \multicolumn{2}{|c|}{ Normal distribution } & \multicolumn{2}{|c|}{ Heavy-tailed distribution ${ }^{a}$} & \multicolumn{2}{|c|}{ Skewed distribution a } \\
\hline & & $\begin{array}{l}\text { Cochran's } \\
\text { Test }(1 \%)\end{array}$ & k-Test $(0.5 \%)^{c}$ & $\begin{array}{l}\text { Cochran's } \\
\text { Test }(1 \%)\end{array}$ & k-Test $(0.5 \%)$ & $\begin{array}{l}\text { Cochran's } \\
\text { Test }(1 \%)\end{array}$ & k-Test $(0.5 \%)$ \\
\hline \multirow[t]{8}{*}{5} & 3 & $1.0 \%$ & $2.5 \%$ & $5.6 \%$ & $9.7 \%$ & $9.1 \%$ & $14.8 \%$ \\
\hline & 4 & $1.1 \%$ & $2.6 \%$ & $8.3 \%$ & $12.9 \%$ & $12.4 \%$ & $18.3 \%$ \\
\hline & 5 & $1.1 \%$ & $2.8 \%$ & $10.8 \%$ & $15.6 \%$ & $15.1 \%$ & $22.0 \%$ \\
\hline & 6 & $1.2 \%$ & $2.7 \%$ & $12.2 \%$ & $17.5 \%$ & $16.6 \%$ & $24.0 \%$ \\
\hline & 7 & $0.9 \%$ & $2.6 \%$ & $14.0 \%$ & $19.3 \%$ & $18.2 \%$ & $25.4 \%$ \\
\hline & 8 & $0.9 \%$ & $2.5 \%$ & $15.1 \%$ & $20.7 \%$ & $19.6 \%$ & $27.7 \%$ \\
\hline & 9 & $0.9 \%$ & $2.3 \%$ & $16.2 \%$ & $22.3 \%$ & $20.0 \%$ & $28.0 \%$ \\
\hline & 10 & $1.1 \%$ & $2.5 \%$ & $17.0 \%$ & $22.9 \%$ & $21.5 \%$ & $29.5 \%$ \\
\hline \multirow[t]{8}{*}{10} & 3 & $1.0 \%$ & $5.3 \%$ & $11.5 \%$ & $22.7 \%$ & $15.6 \%$ & $30.9 \%$ \\
\hline & 4 & $0.9 \%$ & $4.9 \%$ & $16.0 \%$ & $29.0 \%$ & $20.6 \%$ & $37.7 \%$ \\
\hline & 5 & $0.9 \%$ & $4.8 \%$ & $18.8 \%$ & $33.1 \%$ & $24.4 \%$ & $42.7 \%$ \\
\hline & 6 & $1.1 \%$ & $5.3 \%$ & $21.8 \%$ & $35.8 \%$ & $27.1 \%$ & $45.9 \%$ \\
\hline & 7 & $1.1 \%$ & $5.0 \%$ & $23.6 \%$ & $38.6 \%$ & $29.0 \%$ & $48.1 \%$ \\
\hline & 8 & $1.1 \%$ & $5.2 \%$ & $25.2 \%$ & $41.0 \%$ & $30.5 \%$ & $49.9 \%$ \\
\hline & 9 & $1.0 \%$ & $5.0 \%$ & $27.4 \%$ & $42.5 \%$ & $32.0 \%$ & $52.0 \%$ \\
\hline & 10 & $0.9 \%$ & $4.6 \%$ & $28.3 \%$ & $44.3 \%$ & $33.9 \%$ & $53.6 \%$ \\
\hline \multirow[t]{8}{*}{15} & 3 & $1.1 \%$ & $7.9 \%$ & $16.3 \%$ & $34.7 \%$ & $20.7 \%$ & $45.1 \%$ \\
\hline & 4 & $1.0 \%$ & $7.5 \%$ & $22.0 \%$ & $41.8 \%$ & $27.3 \%$ & $53.1 \%$ \\
\hline & 5 & $0.9 \%$ & $7.6 \%$ & $25.7 \%$ & $47.2 \%$ & $31.1 \%$ & $57.9 \%$ \\
\hline & 6 & $0.9 \%$ & $7.9 \%$ & $28.6 \%$ & $50.4 \%$ & $34.6 \%$ & $61.2 \%$ \\
\hline & 7 & $0.9 \%$ & $7.7 \%$ & $31.8 \%$ & $53.8 \%$ & $37.6 \%$ & $64.8 \%$ \\
\hline & 8 & $1.1 \%$ & $7.5 \%$ & $33.8 \%$ & $55.6 \%$ & $39.4 \%$ & $66.4 \%$ \\
\hline & 9 & $1.1 \%$ & $7.5 \%$ & $35.4 \%$ & $57.9 \%$ & $41.2 \%$ & $68.2 \%$ \\
\hline & 10 & $0.9 \%$ & $7.5 \%$ & $37.9 \%$ & $59.8 \%$ & $42.5 \%$ & $69.2 \%$ \\
\hline
\end{tabular}

a The heavy-tailed distribution is a t-distribution with four degrees of freedom. The skewed distribution is an exponential distribution.

b Cochran's Test should show statistical significance approximately $1 \%$ of the time. Unlike the k-statistic its significance level does account for testing multiplicity.

c The k-test should show statistical significance approximately $0.5 \%{ }^{*}$ Number of Labs of the time. This statistic is sometimes referred to as Mandel's $\mathrm{k}$ and the nominal significance level $(0.5 \%$ in this instance) does not account for testing multiplicity.

\section{SIMULATION RESULTS AND IMPLICATIONS}

\subsection{Cochran's Test and the k-statistic}

The first simulation examines the sensitivity of Cochran's Test and the k-statistic to non-normality. As discussed above, in principle, the k-statistic is statistically equivalent to Cochran's Test, but it is implemented with a criterion that is much more likely to eliminate laboratories as outliers, since, as previously noted, its critical values do not take account of multiple testing.

Simulation results are given in Table 2 for situations commonly encountered in collaborative studies and include the two non-normal distributions discussed above. The results in the table confirm the sensitivity of Cochran's Test to non-normality, since much more than $1 \%$ of the results are significant when the underlying distribution is not normal. The results in Table 2 are also consistent with the results given in CONOVER et al. (5), but are more applicable to a collaborative study setting. The results also confirm that the k-statistic in ASTM E691-2014 is much more prone to falsely identify outliers than Cochran's Test as discussed from a more theoretical perspective above. Looking at the k-statistic results in the normal distribution case illustrates the impact of not correcting for testing multiplicity, since the actual level of the test is approximately $p \times 0.5 \%$ when the data are normally distributed.

\subsection{Levene's test simulations}

Simulation results of the four different implementations of Levene's Test are given in Table 3. As shown in Table 3, only L 0 is both robust and close to the nominal significance level. It was further concluded in CONOVER et al. (5) that procedures such as L0 and Lmed were among the best choices as regards statistical power. On the basis of these results, the L0 approach was adapted to derive an alternative to Cochran's Test. Again we emphasize that, when employing L0, the median is not deleted from the actual data set, the $D_{i}$ associated with the median is only omitted from the outlier detection calculation.

\subsection{Alternative approach to Cochran's Test}

As noted above, Levene's Test only tests whether there are differences in variances, not whether a laboratory has excessively large within-laboratory variance. We adapt Levene's Test to determine if the laboratory with the largest variance should be judged an outlier using a two-step approach. 
Table 3. Simulation of four different implementations of Levene's Test .

\begin{tabular}{|c|c|c|c|c|c|c|c|c|c|c|c|c|c|}
\hline \multirow{3}{*}{$\begin{array}{l}\text { No. } \\
\text { Labs }\end{array}$} & \multirow{3}{*}{$\begin{array}{l}\text { No. } \\
\text { Reps }\end{array}$} & \multicolumn{12}{|c|}{ Nominal significance level } \\
\hline & & \multicolumn{3}{|c|}{ L2 } & \multicolumn{3}{|c|}{ L1 } & \multicolumn{3}{|c|}{ Lmed (Median kept) } & \multicolumn{3}{|c|}{ LO (Median omitted $\mathrm{n}$ reps odd) } \\
\hline & & $5 \%$ & $2 \%$ & $1 \%$ & $5 \%$ & $2 \%$ & $1 \%$ & $5 \%$ & $2 \%$ & $1 \%$ & $5 \%$ & $2 \%$ & $1 \%$ \\
\hline \multicolumn{14}{|c|}{ Normal Distribution } \\
\hline \multirow[t]{8}{*}{5} & 3 & $0.8 \%$ & $0.0 \%$ & $0.0 \%$ & $12.3 \%$ & $3.7 \%$ & $1.2 \%$ & $0.0 \%$ & $0.0 \%$ & $0.0 \%$ & $3.4 \%$ & $1.7 \%$ & $0.8 \%$ \\
\hline & 4 & $7.8 \%$ & $4.2 \%$ & $2.9 \%$ & $11.7 \%$ & $6.1 \%$ & $4.0 \%$ & $6.7 \%$ & $3.6 \%$ & $2.2 \%$ & $6.7 \%$ & $3.6 \%$ & $2.2 \%$ \\
\hline & 5 & $7.5 \%$ & $3.6 \%$ & $2.1 \%$ & $9.6 \%$ & $4.7 \%$ & $2.5 \%$ & $0.5 \%$ & $0.1 \%$ & $0.0 \%$ & $3.2 \%$ & $1.4 \%$ & $0.8 \%$ \\
\hline & 6 & $7.1 \%$ & $3.2 \%$ & $2.0 \%$ & $9.2 \%$ & $4.3 \%$ & $2.5 \%$ & $3.7 \%$ & $1.6 \%$ & $1.0 \%$ & $3.7 \%$ & $1.6 \%$ & $1.0 \%$ \\
\hline & 7 & $6.6 \%$ & $2.7 \%$ & $1.4 \%$ & $7.8 \%$ & $3.3 \%$ & $1.8 \%$ & $1.0 \%$ & $0.2 \%$ & $0.0 \%$ & $3.8 \%$ & $1.5 \%$ & $0.8 \%$ \\
\hline & 8 & $6.2 \%$ & $2.6 \%$ & $1.5 \%$ & $7.9 \%$ & $3.6 \%$ & $1.9 \%$ & $3.2 \%$ & $1.1 \%$ & $0.5 \%$ & $3.2 \%$ & $1.1 \%$ & $0.5 \%$ \\
\hline & 9 & $5.7 \%$ & $2.3 \%$ & $1.2 \%$ & $7.0 \%$ & $2.8 \%$ & $1.5 \%$ & $1.3 \%$ & $0.4 \%$ & $0.1 \%$ & $3.8 \%$ & $1.4 \%$ & $0.7 \%$ \\
\hline & 10 & $6.1 \%$ & $2.5 \%$ & $1.3 \%$ & $6.9 \%$ & $3.0 \%$ & $1.6 \%$ & $3.1 \%$ & $1.1 \%$ & $0.6 \%$ & $3.1 \%$ & $1.1 \%$ & $0.6 \%$ \\
\hline \multirow[t]{8}{*}{10} & 3 & $17.0 \%$ & $1.9 \%$ & $0.1 \%$ & $25.6 \%$ & $11.6 \%$ & $5.9 \%$ & $0.0 \%$ & $0.0 \%$ & $0.0 \%$ & $2.5 \%$ & $1.0 \%$ & $0.6 \%$ \\
\hline & 4 & $12.2 \%$ & $6.5 \%$ & $4.2 \%$ & $16.5 \%$ & $8.9 \%$ & $5.3 \%$ & $6.2 \%$ & $3.0 \%$ & $1.9 \%$ & $6.2 \%$ & $3.0 \%$ & $1.9 \%$ \\
\hline & 5 & $10.4 \%$ & $5.2 \%$ & $3.2 \%$ & $12.6 \%$ & $6.5 \%$ & $3.5 \%$ & $0.2 \%$ & $0.0 \%$ & $0.0 \%$ & $2.8 \%$ & $1.2 \%$ & $0.6 \%$ \\
\hline & 6 & $9.4 \%$ & $4.7 \%$ & $2.7 \%$ & $10.8 \%$ & $5.0 \%$ & $2.8 \%$ & $2.9 \%$ & $1.2 \%$ & $0.7 \%$ & $2.9 \%$ & $1.2 \%$ & $0.7 \%$ \\
\hline & 7 & $8.5 \%$ & $4.2 \%$ & $2.4 \%$ & $10.0 \%$ & $4.6 \%$ & $2.7 \%$ & $0.6 \%$ & $0.2 \%$ & $0.1 \%$ & $3.5 \%$ & $1.3 \%$ & $0.8 \%$ \\
\hline & 8 & $8.1 \%$ & $3.7 \%$ & $2.3 \%$ & $9.2 \%$ & $4.2 \%$ & $2.4 \%$ & $2.5 \%$ & $1.0 \%$ & $0.4 \%$ & $2.5 \%$ & $1.0 \%$ & $0.4 \%$ \\
\hline & 9 & $7.6 \%$ & $3.4 \%$ & $1.9 \%$ & $8.4 \%$ & $3.7 \%$ & $2.1 \%$ & $1.0 \%$ & $0.3 \%$ & $0.2 \%$ & $3.7 \%$ & $1.5 \%$ & $0.8 \%$ \\
\hline & 10 & $7.2 \%$ & $3.3 \%$ & $1.7 \%$ & $8.0 \%$ & $3.7 \%$ & $2.1 \%$ & $2.7 \%$ & $1.0 \%$ & $0.5 \%$ & $2.7 \%$ & $1.0 \%$ & $0.5 \%$ \\
\hline \multirow[t]{8}{*}{15} & 3 & $37.9 \%$ & $11.9 \%$ & $3.3 \%$ & $38.0 \%$ & $19.9 \%$ & $11.7 \%$ & $0.0 \%$ & $0.0 \%$ & $0.0 \%$ & $1.8 \%$ & $0.7 \%$ & $0.3 \%$ \\
\hline & 4 & $17.7 \%$ & $9.4 \%$ & $6.0 \%$ & $21.0 \%$ & $11.7 \%$ & $7.7 \%$ & $6.1 \%$ & $3.2 \%$ & $2.1 \%$ & $6.1 \%$ & $3.2 \%$ & $2.1 \%$ \\
\hline & 5 & $13.7 \%$ & $7.2 \%$ & $4.5 \%$ & $15.4 \%$ & $8.1 \%$ & $5.2 \%$ & $0.1 \%$ & $0.0 \%$ & $0.0 \%$ & $2.3 \%$ & $0.9 \%$ & $0.4 \%$ \\
\hline & 6 & $12.0 \%$ & $6.1 \%$ & $3.8 \%$ & $13.0 \%$ & $6.8 \%$ & $3.9 \%$ & $2.5 \%$ & $1.1 \%$ & $0.6 \%$ & $2.5 \%$ & $1.1 \%$ & $0.6 \%$ \\
\hline & 7 & $10.6 \%$ & $5.0 \%$ & $2.8 \%$ & $11.4 \%$ & $5.2 \%$ & $3.0 \%$ & $0.3 \%$ & $0.1 \%$ & $0.0 \%$ & $3.1 \%$ & $1.3 \%$ & $0.5 \%$ \\
\hline & 8 & $9.5 \%$ & $4.9 \%$ & $2.8 \%$ & $10.2 \%$ & $4.9 \%$ & $2.8 \%$ & $2.3 \%$ & $0.8 \%$ & $0.4 \%$ & $2.3 \%$ & $0.8 \%$ & $0.4 \%$ \\
\hline & 9 & $9.1 \%$ & $4.4 \%$ & $2.6 \%$ & $9.5 \%$ & $4.4 \%$ & $2.5 \%$ & $0.9 \%$ & $0.2 \%$ & $0.1 \%$ & $3.8 \%$ & $1.4 \%$ & $0.8 \%$ \\
\hline & 10 & $8.5 \%$ & $3.7 \%$ & $2.2 \%$ & $8.8 \%$ & $4.2 \%$ & $2.4 \%$ & $2.3 \%$ & $0.8 \%$ & $0.4 \%$ & $2.3 \%$ & $0.8 \%$ & $0.4 \%$ \\
\hline \multicolumn{14}{|c|}{ Heavy-tailed distribution ( $t$ with 4 degrees of freedom) } \\
\hline \multirow[t]{8}{*}{5} & 3 & $5.0 \%$ & $0.0 \%$ & $0.0 \%$ & $23.9 \%$ & $11.3 \%$ & $5.9 \%$ & $0.0 \%$ & $0.0 \%$ & $0.0 \%$ & $4.6 \%$ & $2.1 \%$ & $1.0 \%$ \\
\hline & 4 & $7.0 \%$ & $3.7 \%$ & $2.6 \%$ & $17.0 \%$ & $8.1 \%$ & $4.6 \%$ & $6.0 \%$ & $3.2 \%$ & $2.1 \%$ & $6.0 \%$ & $3.2 \%$ & $2.1 \%$ \\
\hline & 5 & $7.0 \%$ & $3.4 \%$ & $1.9 \%$ & $13.3 \%$ & $6.2 \%$ & $3.5 \%$ & $0.5 \%$ & $0.1 \%$ & $0.1 \%$ & $3.7 \%$ & $1.4 \%$ & $0.7 \%$ \\
\hline & 6 & $6.7 \%$ & $3.1 \%$ & $1.7 \%$ & $12.0 \%$ & $5.7 \%$ & $3.2 \%$ & $3.5 \%$ & $1.6 \%$ & $0.8 \%$ & $3.5 \%$ & $1.6 \%$ & $0.8 \%$ \\
\hline & 7 & $6.2 \%$ & $2.6 \%$ & $1.5 \%$ & $10.7 \%$ & $4.7 \%$ & $2.7 \%$ & $1.7 \%$ & $0.5 \%$ & $0.2 \%$ & $4.3 \%$ & $1.9 \%$ & $1.0 \%$ \\
\hline & 8 & $5.6 \%$ & $2.4 \%$ & $1.2 \%$ & $9.9 \%$ & $4.4 \%$ & $2.5 \%$ & $3.1 \%$ & $1.2 \%$ & $0.6 \%$ & $3.1 \%$ & $1.2 \%$ & $0.6 \%$ \\
\hline & 9 & $5.4 \%$ & $2.1 \%$ & $1.0 \%$ & $9.4 \%$ & $4.1 \%$ & $2.2 \%$ & $2.1 \%$ & $0.5 \%$ & $0.3 \%$ & $4.4 \%$ & $1.5 \%$ & $0.7 \%$ \\
\hline & 10 & $5.3 \%$ & $2.3 \%$ & $1.0 \%$ & $9.2 \%$ & $4.2 \%$ & $2.3 \%$ & $3.6 \%$ & $1.4 \%$ & $0.8 \%$ & $3.6 \%$ & $1.4 \%$ & $0.8 \%$ \\
\hline \multirow[t]{8}{*}{10} & 3 & $42.6 \%$ & $16.0 \%$ & $4.5 \%$ & $49.5 \%$ & $32.1 \%$ & $22.6 \%$ & $0.0 \%$ & $0.0 \%$ & $0.0 \%$ & $4.5 \%$ & $2.1 \%$ & $1.2 \%$ \\
\hline & 4 & $15.9 \%$ & $6.9 \%$ & $4.5 \%$ & $32.2 \%$ & $19.1 \%$ & $13.1 \%$ & $6.6 \%$ & $3.6 \%$ & $2.3 \%$ & $6.6 \%$ & $3.6 \%$ & $2.3 \%$ \\
\hline & 5 & $11.3 \%$ & $5.6 \%$ & $3.4 \%$ & $23.5 \%$ & $13.3 \%$ & $8.6 \%$ & $0.6 \%$ & $0.1 \%$ & $0.0 \%$ & $4.1 \%$ & $1.7 \%$ & $1.0 \%$ \\
\hline & 6 & $10.2 \%$ & $5.1 \%$ & $3.2 \%$ & $20.2 \%$ & $11.1 \%$ & $7.0 \%$ & $3.7 \%$ & $1.4 \%$ & $0.8 \%$ & $3.7 \%$ & $1.4 \%$ & $0.8 \%$ \\
\hline & 7 & $8.8 \%$ & $4.5 \%$ & $2.8 \%$ & $17.3 \%$ & $9.0 \%$ & $5.6 \%$ & $1.4 \%$ & $0.4 \%$ & $0.2 \%$ & $4.6 \%$ & $1.9 \%$ & $1.0 \%$ \\
\hline & 8 & $8.2 \%$ & $4.0 \%$ & $2.5 \%$ & $15.2 \%$ & $7.5 \%$ & $4.7 \%$ & $2.9 \%$ & $1.2 \%$ & $0.6 \%$ & $2.9 \%$ & $1.2 \%$ & $0.6 \%$ \\
\hline & 9 & $7.4 \%$ & $3.4 \%$ & $2.1 \%$ & $13.8 \%$ & $7.0 \%$ & $4.3 \%$ & $1.9 \%$ & $0.6 \%$ & $0.3 \%$ & $4.5 \%$ & $1.8 \%$ & $1.0 \%$ \\
\hline & 10 & $7.1 \%$ & $3.4 \%$ & $2.0 \%$ & $12.8 \%$ & $6.7 \%$ & $3.9 \%$ & $3.3 \%$ & $1.3 \%$ & $0.7 \%$ & $3.3 \%$ & $1.3 \%$ & $0.7 \%$ \\
\hline \multirow[t]{8}{*}{15} & 3 & $71.4 \%$ & $46.1 \%$ & $28.2 \%$ & $68.0 \%$ & $51.6 \%$ & $40.5 \%$ & $0.0 \%$ & $0.0 \%$ & $0.0 \%$ & $4.4 \%$ & $2.2 \%$ & $1.3 \%$ \\
\hline & 4 & $31.4 \%$ & $13.2 \%$ & $7.5 \%$ & $45.2 \%$ & $30.0 \%$ & $22.1 \%$ & $7.0 \%$ & $3.7 \%$ & $2.6 \%$ & $7.0 \%$ & $3.7 \%$ & $2.6 \%$ \\
\hline & 5 & $17.0 \%$ & $8.3 \%$ & $4.9 \%$ & $32.4 \%$ & $20.1 \%$ & $13.8 \%$ & $0.6 \%$ & $0.1 \%$ & $0.1 \%$ & $4.3 \%$ & $1.9 \%$ & $1.1 \%$ \\
\hline & 6 & $13.4 \%$ & $6.7 \%$ & $4.1 \%$ & $27.0 \%$ & $16.4 \%$ & $11.1 \%$ & $3.5 \%$ & $1.6 \%$ & $1.0 \%$ & $3.5 \%$ & $1.6 \%$ & $1.0 \%$ \\
\hline & 7 & $11.7 \%$ & $6.0 \%$ & $3.6 \%$ & $23.2 \%$ & $13.3 \%$ & $8.9 \%$ & $1.4 \%$ & $0.5 \%$ & $0.2 \%$ & $4.9 \%$ & $2.1 \%$ & $1.2 \%$ \\
\hline & 8 & $9.7 \%$ & $4.8 \%$ & $3.1 \%$ & $20.1 \%$ & $11.1 \%$ & $7.2 \%$ & $2.8 \%$ & $1.2 \%$ & $0.7 \%$ & $2.8 \%$ & $1.2 \%$ & $0.7 \%$ \\
\hline & 9 & $9.0 \%$ & $4.5 \%$ & $2.7 \%$ & $17.9 \%$ & $9.8 \%$ & $6.2 \%$ & $1.8 \%$ & $0.8 \%$ & $0.4 \%$ & $4.6 \%$ & $1.9 \%$ & $1.2 \%$ \\
\hline & 10 & $8.6 \%$ & $4.3 \%$ & $2.7 \%$ & $17.0 \%$ & $9.3 \%$ & $6.0 \%$ & $3.4 \%$ & $1.2 \%$ & $0.5 \%$ & $3.4 \%$ & $1.2 \%$ & $0.5 \%$ \\
\hline
\end{tabular}


Table 3. Contd.

\begin{tabular}{|c|c|c|c|c|c|c|c|c|c|c|c|c|c|}
\hline \multirow{3}{*}{$\begin{array}{l}\text { No. } \\
\text { Labs }\end{array}$} & \multirow{3}{*}{$\begin{array}{l}\text { No. } \\
\text { Reps }\end{array}$} & \multicolumn{12}{|c|}{ Nominal significance level } \\
\hline & & \multicolumn{3}{|c|}{$\mathrm{L} 2$} & \multicolumn{3}{|c|}{ L1 } & \multicolumn{3}{|c|}{ Lmed (Median kept) } & \multicolumn{3}{|c|}{ L0 (Median omitted $\mathrm{n}$ reps odd) } \\
\hline & & $5 \%$ & $2 \%$ & $1 \%$ & $5 \%$ & $2 \%$ & $1 \%$ & $5 \%$ & $2 \%$ & $1 \%$ & $5 \%$ & $2 \%$ & $1 \%$ \\
\hline \multicolumn{14}{|c|}{ Skewed Distribution (exponential) } \\
\hline \multirow[t]{8}{*}{5} & 3 & $8.2 \%$ & $0.0 \%$ & $0.0 \%$ & $42.8 \%$ & $24.9 \%$ & $14.4 \%$ & $0.0 \%$ & $0.0 \%$ & $0.0 \%$ & $3.4 \%$ & $1.6 \%$ & $1.0 \%$ \\
\hline & 4 & $12.1 \%$ & $7.4 \%$ & $5.6 \%$ & $33.2 \%$ & $18.3 \%$ & $11.2 \%$ & $10.8 \%$ & $6.7 \%$ & $4.9 \%$ & $10.8 \%$ & $6.7 \%$ & $4.9 \%$ \\
\hline & 5 & $13.4 \%$ & $8.2 \%$ & $5.6 \%$ & $29.9 \%$ & $17.7 \%$ & $12.1 \%$ & $1.2 \%$ & $0.3 \%$ & $0.2 \%$ & $4.7 \%$ & $2.3 \%$ & $1.3 \%$ \\
\hline & 6 & $13.0 \%$ & $7.2 \%$ & $4.8 \%$ & $28.6 \%$ & $17.5 \%$ & $12.0 \%$ & $6.0 \%$ & $3.2 \%$ & $1.9 \%$ & $6.0 \%$ & $3.2 \%$ & $1.9 \%$ \\
\hline & 7 & $11.9 \%$ & $6.3 \%$ & $4.0 \%$ & $27.5 \%$ & $17.1 \%$ & $11.8 \%$ & $2.6 \%$ & $1.2 \%$ & $0.6 \%$ & $5.2 \%$ & $2.4 \%$ & $1.6 \%$ \\
\hline & 8 & $11.4 \%$ & $5.9 \%$ & $3.6 \%$ & $27.6 \%$ & $17.2 \%$ & $12.0 \%$ & $5.3 \%$ & $2.6 \%$ & $1.4 \%$ & $5.3 \%$ & $2.6 \%$ & $1.4 \%$ \\
\hline & 9 & $9.9 \%$ & $5.1 \%$ & $3.1 \%$ & $27.0 \%$ & $16.8 \%$ & $11.6 \%$ & $2.9 \%$ & $1.2 \%$ & $0.7 \%$ & $5.1 \%$ & $2.2 \%$ & $1.4 \%$ \\
\hline & 10 & $9.3 \%$ & $4.8 \%$ & $3.0 \%$ & $27.0 \%$ & $16.6 \%$ & $11.7 \%$ & $4.9 \%$ & $2.2 \%$ & $1.3 \%$ & $4.9 \%$ & $2.2 \%$ & $1.3 \%$ \\
\hline \multirow[t]{8}{*}{10} & 3 & $59.9 \%$ & $23.9 \%$ & $5.7 \%$ & $75.8 \%$ & $60.2 \%$ & $47.6 \%$ & $0.0 \%$ & $0.0 \%$ & $0.0 \%$ & $2.6 \%$ & $1.2 \%$ & $0.9 \%$ \\
\hline & 4 & $22.0 \%$ & $13.0 \%$ & $9.3 \%$ & $61.7 \%$ & $44.5 \%$ & $33.2 \%$ & $12.2 \%$ & $7.7 \%$ & $5.6 \%$ & $12.2 \%$ & $7.7 \%$ & $5.6 \%$ \\
\hline & 5 & $18.6 \%$ & $11.7 \%$ & $8.5 \%$ & $52.4 \%$ & $36.6 \%$ & $26.8 \%$ & $1.1 \%$ & $0.4 \%$ & $0.2 \%$ & $4.7 \%$ & $2.3 \%$ & $1.3 \%$ \\
\hline & 6 & $17.0 \%$ & $10.5 \%$ & $7.5 \%$ & $48.9 \%$ & $33.8 \%$ & $25.6 \%$ & $5.9 \%$ & $2.9 \%$ & $1.8 \%$ & $5.9 \%$ & $2.9 \%$ & $1.8 \%$ \\
\hline & 7 & $15.1 \%$ & $9.3 \%$ & $6.5 \%$ & $45.6 \%$ & $31.4 \%$ & $23.4 \%$ & $2.2 \%$ & $0.8 \%$ & $0.4 \%$ & $5.1 \%$ & $2.4 \%$ & $1.4 \%$ \\
\hline & 8 & $13.4 \%$ & $7.8 \%$ & $5.4 \%$ & $44.4 \%$ & $30.5 \%$ & $22.6 \%$ & $4.6 \%$ & $2.1 \%$ & $1.2 \%$ & $4.6 \%$ & $2.1 \%$ & $1.2 \%$ \\
\hline & 9 & $12.5 \%$ & $7.1 \%$ & $4.6 \%$ & $42.9 \%$ & $29.5 \%$ & $22.6 \%$ & $2.8 \%$ & $1.1 \%$ & $0.6 \%$ & $5.3 \%$ & $2.3 \%$ & $1.3 \%$ \\
\hline & 10 & $12.3 \%$ & $6.7 \%$ & $4.4 \%$ & $41.9 \%$ & $29.3 \%$ & $22.2 \%$ & $4.7 \%$ & $2.1 \%$ & $1.1 \%$ & $4.7 \%$ & $2.1 \%$ & $1.1 \%$ \\
\hline \multirow[t]{8}{*}{15} & 3 & $86.7 \%$ & $63.8 \%$ & $41.2 \%$ & $91.2 \%$ & $82.0 \%$ & $73.2 \%$ & $0.0 \%$ & $0.0 \%$ & $0.0 \%$ & $2.2 \%$ & $1.1 \%$ & $0.7 \%$ \\
\hline & 4 & $37.9 \%$ & $18.7 \%$ & $13.1 \%$ & $79.0 \%$ & $64.9 \%$ & $54.0 \%$ & $12.1 \%$ & $7.9 \%$ & $5.7 \%$ & $12.1 \%$ & $7.9 \%$ & $5.7 \%$ \\
\hline & 5 & $23.7 \%$ & $14.9 \%$ & $10.8 \%$ & $68.6 \%$ & $53.1 \%$ & $42.5 \%$ & $0.9 \%$ & $0.3 \%$ & $0.2 \%$ & $4.4 \%$ & $2.1 \%$ & $1.2 \%$ \\
\hline & 6 & $20.7 \%$ & $12.9 \%$ & $9.4 \%$ & $63.8 \%$ & $48.2 \%$ & $38.5 \%$ & $5.5 \%$ & $2.9 \%$ & $1.8 \%$ & $5.5 \%$ & $2.9 \%$ & $1.8 \%$ \\
\hline & 7 & $18.2 \%$ & $11.4 \%$ & $8.3 \%$ & $60.6 \%$ & $45.3 \%$ & $35.8 \%$ & $1.8 \%$ & $0.6 \%$ & $0.3 \%$ & $5.3 \%$ & $2.3 \%$ & $1.2 \%$ \\
\hline & 8 & $16.2 \%$ & $9.6 \%$ & $6.6 \%$ & $58.3 \%$ & $43.4 \%$ & $34.4 \%$ & $4.6 \%$ & $2.1 \%$ & $1.3 \%$ & $4.6 \%$ & $2.1 \%$ & $1.3 \%$ \\
\hline & 9 & $14.7 \%$ & $8.6 \%$ & $5.9 \%$ & $57.0 \%$ & $42.7 \%$ & $33.5 \%$ & $2.7 \%$ & $1.1 \%$ & $0.6 \%$ & $5.3 \%$ & $2.6 \%$ & $1.5 \%$ \\
\hline & 10 & $13.2 \%$ & $7.8 \%$ & $5.3 \%$ & $54.9 \%$ & $40.9 \%$ & $31.9 \%$ & $4.5 \%$ & $2.0 \%$ & $1.3 \%$ & $4.5 \%$ & $2.0 \%$ & $1.3 \%$ \\
\hline
\end{tabular}

1. The L0 version of Levene's Test is carried out using a nominal significance level of $\alpha=0.02$. The 0.02 value was chosen, both because the L0 test is often a little conservative in its actual level and because both step 1 and step 2 must be significant to declare a laboratory an outlier.

2. The laboratory with the largest average deviations is compared via a linear contrast to the average of the remaining laboratories. Statistical significance is judged using a nominal one-sided $\alpha=0.01 /$ (number of laboratories). The test is one-sided because we are only interested in the laboratory with the largest deviations. The division by the number of laboratories is a Bonferroni adjustment to account for multiple testing. It is necessary to account for multiple testing because it is not known a priori which laboratory will have the largest within-laboratory variation. If this test is significant, then the laboratory with the largest average deviation is declared an outlier; otherwise, no laboratory is identified as an outlier. ${ }^{1}$

Simulation results of this approach are shown in Table 4. It can be seen that this approach is robust to the deviations from normality considered here and close to the stated $1 \%$ false rejection rate.

1 A different alternative approach would be to use step 2 alone. Simulations not shown in this manuscript showed that using step 2 alone, though better than Cochran's Test, is much less robust to nonnormality than using the combination of step 1 and 2 together.
To illustrate this approach, consider the data in Figure 2. There are 10 laboratories and three replicates per laboratory. As can be seen in Figure 2, Laboratory 10 has the largest spread amongst its replicates. Cochran's Test statistic is

$$
c=\frac{0.0650^{2}}{0.0015^{2}+\ldots+0.0650^{2}}=0.736>0.536,
$$

where 0.536 is the critical value.

Thus Cochran's Test is statistically significant and, using that test, Laboratory 10 would be dropped from the analysis.

To apply the alternative approach, the median value within each laboratory is calculated, along with the absolute deviation of each replicate from the median as shown in Table 5. Since the number of replicates is odd, the median value is not included in the calculation of the absolute deviations and is also not included in the one-way analysis of variance.

The $\mathrm{p}$-value from the overall analysis of variance is $p=0.11>0.02$, and, therefore, is not statistically significant. It would be unnecessary in this case, but, for illustration, we also calculate the comparison of the absolute deviations of Laboratory 10 to the others as a linear contrast in the analysis of variance: 


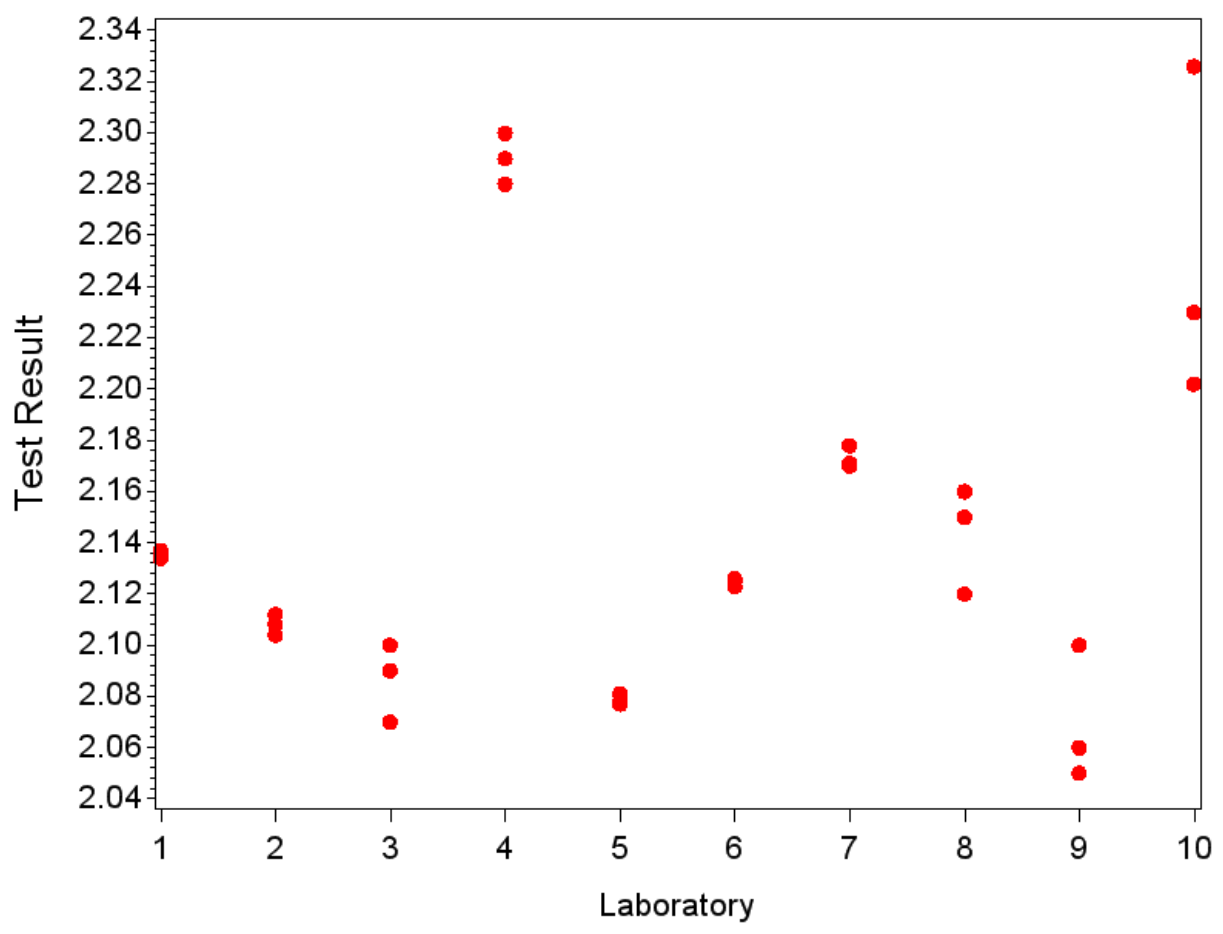

Figure 2. Graph of example data from collaborative study.

$\overline{D_{10}}-\frac{1}{9} \sum_{i=1}^{9} \overline{D_{i}}$.

The nominal p-value is 0.0023 and is compared to $0.02 / 10=0.002$, and, thus, the linear contrast (step 2 above) is also not statistically significant (though it is "close") ${ }^{2}$. If either of the two stages is not statistically significant, the laboratory is not deemed an outlier and, therefore, by these criteria, Laboratory 10 is not declared an outlier.

The effect of keeping $v s$. dropping Laboratory 10 is shown in Table 6. Since the laboratory is kept or dropped based on its within-laboratory variability, one would expect that keeping or dropping the laboratory would most typically have a large effect on the estimated method repeatability (r), as is shown in Table 6.

In utilizing this approach in the analysis of numerous collaborative studies, in the author's opinion, the method has generally performed quite reasonably. However, one shortcoming was observed in the occasionally encountered case of a single-point outlier within a laboratory.

To illustrate the difficulty with single-point outliers, consider the data shown in Figure 3. From the graph, one of the replicates in Laboratory 2 appears to be a clear outlier. Carrying out the analysis of variance as described above gives $p=0.47$. So the laboratory would not be flagged as an outlier. Note that, since the overall analysis of variance is not statistically significant, this is a shortcoming

2 The value of 0.02 in the equation is chosen because most statistical programs provide the $\mathrm{p}$-values as two-sided $\mathrm{p}$-values. However, this is a one-sided test and 0.02 for a two-sided test is equivalent to 0.01 for a one-sided test. of Levene's Test in general and not this approach alone. Particularly with small numbers of replicates per laboratory, this type of outlier is not readily detected by Levene's Test with $\tilde{x}$ defined to be the median, so, when applied, the practitioner must be watchful for these types of outliers and manually eliminate them. Such outliers can have a dramatic effect on the calculated repeatability. In this instance, the repeatability standard deviation is 0.21 with the outlier excluded (dropping only the single outlying point and keeping the two other values from that laboratory) and the repeatability standard deviation is 0.55 with the outlier included.

\section{SUMMARY AND CONCLUSIONS}

Estimating the variability of an analytical method is an important component of characterizing the quality of the analytical method, and is commonly carried out through collaborative studies. As illustrated above, outlier identification can have a large effect on the estimation of the variability associated with the analytical method, since the estimated variability can be quite different depending on whether a laboratory is or is not identified as an outlier. Commonly followed guides for the analysis of collaborative studies such as ISO 5725-2, ASTM E691-2014, and IUPAC's "Harmonized statistical procedure" recommend Cochran's Test or the k-statistic (sometimes also called Mandel's k) as the tool for the identification of laboratories with excessively large within-laboratory variation. However, as shown above, these tests are very strongly affected by deviations from normality. Moreover, it was shown that deviations from normality that are very difficult to detect analytically can have a very substantial effect on Cochran's Test and the k-statistic and that both too readily identify outliers. 
Table 4. Simulation of alternative approach to identify laboratories with large intra-laboratory variability.

\begin{tabular}{|c|c|c|c|c|}
\hline No. Labs & No. Reps & Normal & Heavy-tailed $^{a}$ & Skewed $^{b}$ \\
\hline \multirow[t]{8}{*}{5} & 3 & $1.1 \%$ & $1.6 \%$ & $1.3 \%$ \\
\hline & 4 & $2.7 \%$ & $2.7 \%$ & $5.8 \%$ \\
\hline & 5 & $0.9 \%$ & $1.1 \%$ & $1.8 \%$ \\
\hline & 6 & $1.1 \%$ & $1.4 \%$ & $2.6 \%$ \\
\hline & 7 & $0.9 \%$ & $1.4 \%$ & $1.9 \%$ \\
\hline & 8 & $0.7 \%$ & $0.9 \%$ & $2.0 \%$ \\
\hline & 9 & $0.8 \%$ & $1.2 \%$ & $1.6 \%$ \\
\hline & 10 & $0.8 \%$ & $1.2 \%$ & $1.6 \%$ \\
\hline \multirow[t]{8}{*}{10} & 3 & $0.8 \%$ & $1.9 \%$ & $1.1 \%$ \\
\hline & 4 & $2.1 \%$ & $3.2 \%$ & $6.5 \%$ \\
\hline & 5 & $0.7 \%$ & $1.4 \%$ & $1.9 \%$ \\
\hline & 6 & $0.7 \%$ & $1.2 \%$ & $2.4 \%$ \\
\hline & 7 & $0.9 \%$ & $1.6 \%$ & $2.0 \%$ \\
\hline & 8 & $0.6 \%$ & $0.9 \%$ & $1.6 \%$ \\
\hline & 9 & $0.8 \%$ & $1.4 \%$ & $1.6 \%$ \\
\hline & 10 & $0.5 \%$ & $1.0 \%$ & $1.5 \%$ \\
\hline \multirow[t]{8}{*}{15} & 3 & $0.5 \%$ & $1.9 \%$ & $0.9 \%$ \\
\hline & 4 & $2.0 \%$ & $3.1 \%$ & $6.5 \%$ \\
\hline & 5 & $0.5 \%$ & $1.6 \%$ & $1.7 \%$ \\
\hline & 6 & $0.7 \%$ & $1.3 \%$ & $2.0 \%$ \\
\hline & 7 & $0.7 \%$ & $1.6 \%$ & $1.7 \%$ \\
\hline & 8 & $0.4 \%$ & $0.9 \%$ & $1.6 \%$ \\
\hline & 9 & $0.7 \%$ & $1.4 \%$ & $1.8 \%$ \\
\hline & 10 & $0.3 \%$ & $0.9 \%$ & $1.5 \%$ \\
\hline
\end{tabular}

a The heavy-tailed distribution is a t-distribution with 4 degrees of freedom.

b The skewed distribution is an exponential.

NOTE: The nominal $\alpha$-level is $1 \%$. The alternative approach consists of LO with the median removed when the number of replicates is odd. The test is then two-steps: 1) An overall F-test is applied with a nominal a-level of $2 \%$. 2) A one-sided Bonferroni-adjusted linear contrast of the lab with the largest intralaboratory variance against the average of the remaining labs using $\alpha=1 \%$. If both steps are statistically significant, the lab is dropped as having excessive intra-laboratory variation.

Table 5. Example data set.

\begin{tabular}{|c|c|c|c|c|c|c|}
\hline Lab & \multicolumn{3}{|c|}{ Reps $^{a}$} & Median & \multicolumn{2}{|c|}{ Dev from median $^{b}$} \\
\hline 1 & 2.134 & 2.136 & 2.137 & 2.136 & 0.002 & 0.001 \\
\hline 2 & 2.104 & 2.108 & 2.112 & 2.108 & 0.004 & 0.004 \\
\hline 3 & 2.070 & 2.090 & 2.100 & 2.090 & 0.020 & 0.010 \\
\hline 4 & 2.280 & 2.290 & 2.300 & 2.290 & 0.010 & 0.010 \\
\hline 5 & 2.077 & 2.078 & 2.081 & 2.078 & 0.001 & 0.003 \\
\hline 6 & 2.123 & 2.125 & 2.126 & 2.125 & 0.002 & 0.001 \\
\hline 7 & 2.170 & 2.171 & 2.178 & 2.171 & 0.001 & 0.007 \\
\hline 8 & 2.120 & 2.150 & 2.160 & 2.150 & 0.030 & 0.010 \\
\hline 9 & 2.050 & 2.060 & 2.100 & 2.060 & 0.010 & 0.040 \\
\hline 10 & 2.202 & 2.230 & 2.326 & 2.230 & 0.028 & 0.096 \\
\hline
\end{tabular}

a The replicates are sorted from smallest to largest within each laboratory.

b The absolute deviation from the median is shown for the smallest and largest replicates.

NOTE: The middle replicate will be the median and the deviation of the middle replicate is always zero and is omitted from the intralaboratory outlier calculations.
Table 6. Comparison of estimated repeatability ( $r$ ) and reproducibility $(R)$ as a percent of the mean with and without Lab 10 included.

\begin{tabular}{l|cccc}
\hline Mean & Number of labs & $\mathrm{r}(\%)$ & $\mathrm{R}(\%)$ \\
\hline \multicolumn{4}{c}{ Cochran's } & \\
2.13 & 9 & $1.7 \%$ & $8.9 \%$ \\
& Alternative Approach (1 lab dropped) labs dropped) \\
2.15 & 10 & $3.1 \%$ & $9.9 \%$ \\
\hline
\end{tabular}

This sensitivity to deviations from normality has long been known and alternative approaches to testing for homogeneity of variance have been previously proposed. Levene's Test is a commonly recommended alternative approach, but the simulations involving it have not included the number of groups (laboratories) normally included in collaborative studies and were extended in this paper to more relevant numbers of groups (laboratories). Four different versions of Levene's Test were evaluated in simulations. The results of the simulations demonstrated that:

1. Levene's Test using either the absolute or squared deviation from the mean (L1 and L2) is not robust to non-normality.

2. When the number of replicates is odd, the absolute deviation from the median (sometimes referred to as the Brown-Forsythe Test, Lmed) is quite conservative unless the median point is deleted for the purpose of the outlier test.

3. The absolute deviation from the median is robust to non-normality and close to the stated $\alpha$-level, if the $D_{i}$ associated with the median point is deleted for the purpose of outlier evaluation when the number of replicates is odd (L0).

4. A shortcoming of L0 was also identified, namely that, particularly with a small number of replicates per laboratory, this test is not sensitive to single-point outliers.

An alternative approach to the identification of laboratories with unusually large within-laboratory variation was adapted from the L0 version of Levene's Test, and shown to be much more robust to deviations from normality than Cochran's Test or the k-statistic.

This alternative approach results in fewer laboratories being unnecessarily eliminated from collaborative studies and, therefore, allows more laboratory results to be included in the calculation of reproducibility.

A limitation to the proposed approach is that this version of Levene's Test is not sensitive to extreme single-point outliers. In a separate investigation not included here, it was found that some alternative approaches could detect singlepoint outliers, but were not robust to non-normality. For example, a similar approach utilizing L1 above does detect the previously shown single-point outlier, but L1 was shown to not be robust to deviations from normality. So it could well be that a trade-off must be made between sensitivity to single-point outliers and robustness to nonnormality. The recommendation to remove obvious singlepoint outliers should be applied conservatively. Generally, 


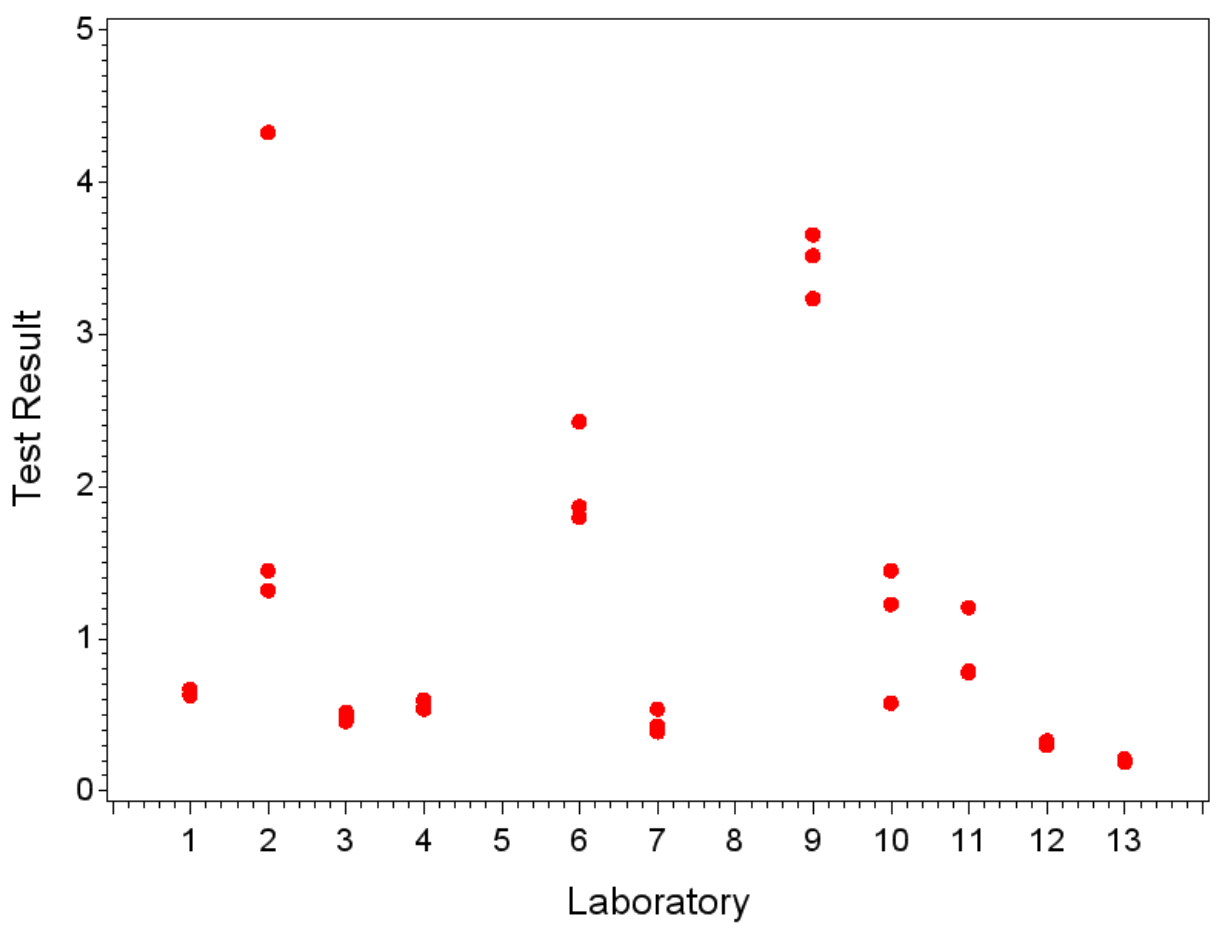

Figure 3. Illustration of collaborative study results with a single-point outlier.

if there is uncertainty as to whether the result is an outlier, then the value should not be removed.

The approach recommended from these results is to use the procedure outlined in this paper together with visual identification and manual removal of obvious single-point outliers.

\section{REFERENCES}

1. International Organization for Standardization (ISO): ISO 5725-2. Accuracy (Trueness and Precision) of Measurement Methods and Results - Part 2: Basic Method for the Determination of Repeatability and Reproducibility of a Standard Measurement Method; ISO, Geneva, Switzerland, 1994.

2. ASTM International: ASTM Standard E691-14. Standard Practice for Conducting an Interlaboratory Study to Determine the Precision of a Test Method; ASTM International, West Conshohocken, PA, USA, 2014.

3. Horwitz, W.: Protocol for the Design, Conduct, and Interpretation of Method-Performance Studies; Pure Appl. Chem. 67 (1995) 331-343.

4. Box, G.E.P.: Non-Normality and Tests on Variances; Biometrika 40 (1953) 318-335. DOI: 10.2307/2333350

5. Conover, W.J., M.E. Johnson, and M.M. Johnson: A Comparative Study of Tests for Homogeneity of Variances, with Applications to the Outer Continental ShelfBidding Data; Technometrics 23 (1981) 351-361. DOI: $10.2307 / 1268225$

6. Levene, H.: Robust Tests for Equality of Variances; in: Contributions to Probability and Statistics, edited by I. Olkin, Stanford University Press, Palo Alto, CA, USA, 1960, pp. 278-292.
7. Brown, M.B. and A.B. Forsythe: Robust Tests for Equality of Variances; J. Am. Stat. Assoc. 69 (1974) 364-367. DOI: $10.2307 / 2285659$

8. Gastwirth, J.L., Y.R. Gel, and W. Miao: The Impact of Levene's Test of Equality of Variances on Statistical Theory and Practice; Stat. Sci. 24 (2009) 343-360. DOI: $10.1214 / 09-S T S 301$

9. Hines, W.G.S. and R.J. O'Hara Hines: Increased Power with Modified Forms of the Levene (Med) Test for Heterogeneity of Variance; Biometrics 56 (2000) 451-454. DOI: 10.1111/j.0006-341X.2000.00451.x

10. Shapiro, S.S. and M.B. Wilk: An Analysis of Variance Test for Normality (Complete Samples): Biometrika 52 (1965) 591-611. DOI: 10.1093/biomet/52.3-4.591

11. Anderson, T.W. and D.A. Darling: Asymptotic Theory of Certain "Goodness-of-Fit" Criteria Based on Stochastic Processes; Ann. Math. Stat. 23 (1952) 193-212. DOI: $10.1214 /$ aoms/1177729437

\section{Corresponding author:}

Michael J. Morton

Altria Client Services LLC

601 E. Jackson

Richmond, VA 23219, USA

Fax: 804-335-2095

E-mail: Michael.J.Morton@altria.com or

SciencePublications@altria.com 